\title{
Tratamento de disfunção temporomandibular com placa oclusal: relato de caso
}

\author{
Temporomandibular dysfunction treatment with occlusal plaque: case report \\ Tratamiento de disfunción temporomandibular con placa oclusal: \\ reporte de caso \\ Igor George da Silva VASCONCELOS ${ }^{1}$ \\ Gisely Naura VENÂNCIO² \\ Franklin Barbosa da SILVA ${ }^{3}$ \\ ${ }^{1}$ Graduado em Odontologia, Universidade Nilton Lins, 69058-030 Manaus-AM, Brasil \\ ${ }^{2}$ Doutoranda em Clínicas Odontológicas - Faculdade São Leopoldo Mandic, 13045-755 Campinas-SP, Brasil \\ ${ }^{3}$ Professor de Prótese Total e Oclusão, Curso de Odontologia, Universidade Nilton Lins, 69058-030 Manaus-AM, Brasil
}

\begin{abstract}
Resumo
O transtorno da articulação temporomandibular (ATM) é uma desordem de etiologia multifatorial que envolve os músculos da mastigação, a ATM, a dentição e estruturas associadas. O objetivo deste trabalho é relatar um caso clínico de tratamento de disfunção temporomandibular por meio da utilização de placa oclusal. A paciente compareceu à clínica da Universidade Nilton Lins, queixando-se de dores orofaciais, na cabeça e nos músculos da mastigação. Ao exame extraoral de palpação da ATM percebeu-se a presença de estalido, crepitação e deslocamento de disco com redução em ambos os lados na abertura bucal. Ao exame intraoral, foi observada mordida 'topo a topo', desvio mandibular na abertura e fechamento, desgastes dentários e ausência do elemento 13. Após análise dos modelos montados em articulador semi-ajustável, o diagnóstico foi de disfunção temporomandibular associada ao bruxismo cêntrico. O tratamento de escolha foi a confecção de placa oclusal superior rígida em resina acrílica termopolimerizável. O procedimento executado foi satisfatório, pois houve redução da intensidade e frequência da dor, com melhora na qualidade de vida da paciente.
\end{abstract}

Descritores: Transtornos da Articulação Temporomandibular; Músculos da Mastigação; Placas Oclusais.

\begin{abstract}
Temporomandibular joint disorder (TMJ) is a multifactorial disorder involving the chewing muscles, the TMJ, the dentition and associated structures. The aim of this study is to report a clinical case of treatment of temporomandibular dysfunction through the use of occlusal splint. The patient attended the Nilton Lins University clinic complaining of orofacial pains in the head and chewing muscles. Extraoral examination of TMJ palpation revealed the presence of crackling, crackling and disc displacement with reduction on both sides of the mouth opening. Intraoral examination, level bite, mandibular deviation at opening and closure, dental wear and absence of element 13 were observed. After analysis of the models mounted in a semi-adjustable articulator, the diagnosis was temporomandibular dysfunction associated with centric bruxism. The treatment of choice was the creation of a rigid upper occlusal splint in thermo-polymerizable acrylic resin. The procedure performed was satisfactory, since there was a reduction in the intensity and frequency of pain, with improvement in the patient's quality of life.
\end{abstract}

Descriptors: Temporomandibular Joint Disorders; Masticatory Muscles; Occlusal Splints.

\section{Resumen}

El trastorno de la articulación temporomandibular (ATM) es un desorden de etiología multifactorial que involucra los músculos de la masticación, la ATM, la dentición y las estructuras asociadas. El objetivo de este trabajo es relatar un caso clínico de tratamiento de disfunción temporomandibular por medio de la utilización de férula oclusal. La paciente asistió a la clínica de la Universidad Nilton Lins, quejándose de dolores orofaciales, en la cabeza y en los músculos de la masticación. En el examen extraoral de palpación de ATM se percibió la presencia de chasquido, crepitación y desplazamiento de disco con reducción en ambos lados en la apertura bucal. En el examen intraoral, se observó una mordedura 'arriba a arriba', desviación mandibular en la apertura y cierre, desgastes dentales y ausencia del elemento 13. Después del análisis de los modelos montados en articulador semi-ajustable, el diagnóstico fue de disfunción temporomandibular asociada al bruxismo céntrico. El tratamiento de elección fue la confección de férula oclusal superior rígida en resina acrílica termopolimerizable. El procedimiento realizado fue satisfactorio, pues hubo reducción de la intensidad y frecuencia del dolor, con mejoría en la calidad de vida de la paciente.

Descriptores: Transtornos de la Articulación Temporomandibular; Músculos Masticadores; Férulas Oclusales.

\section{INTRODUÇÃO}

O termo disfunção temporomandibular (DTM) compreende um conjunto de anormalidades do sistema estomatognático ${ }^{1}$. É especificada como uma forma coletiva que integra vários problemas clínicos referentes à articulação temporomandibular (ATM), músculos da mastigação e estruturas associadas ${ }^{2-4}$. Geralmente o primeiro sintoma que leva o paciente a procurar o tratamento é a dor, podendo ser bilateral ou unilateral, comumente desencadeada por movimentos da mandíbula ou por palpação ${ }^{5}$. Também podemos destacar outros como: ruídos na articulação, dificuldade para abrir e fechar a boca, cefaléia, espasmos, fadiga dos músculos da mastigação, estruturas associadas à cavidade auricular e bruxismo ${ }^{1,6,7}$.

As DTMs são um importante problema na saúde pública, atingindo aproximadamente de $5 \%$ a $12 \%$ da população mundial ${ }^{8,9}$. Com o surgimento de seus sinais e sintomas há uma interferência nas atividades diárias e no funcionamento psicossocial, resultando em uma queda na qualidade de vida do indivíduo ${ }^{1,6,10}$. A sua origem é multifatorial e pode ser desencadeada devido à agitação da vida moderna, estresse, desordens funcionais do sistema mastigatório, má oclusão, atividades posturais inadequadas e fatores emocionais ${ }^{7,10,11}$.
Como formas de tratamentos estão: educação do paciente, auto cuidado, alteração de hábitos comportamentais, medicamentos, atividades fisioterápicas, placas oclusais estabilizadoras, terapia oclusal, tratamento ortodôntico, reabilitação oral, acupuntura, laserterapia, ondas de choques e cirurgia $^{4,9}$. O tratamento conservador é o mais indicado para os casos iniciais de DTMs, por apresentar bons resultados e não ser irreversível ${ }^{4}$. Desta forma, o objetivo do presente trabalho é relatar um caso clínico de tratamento da disfunção temporomandibular por meio da utilização de placa oclusal para a redução da sintomatologia dolorosa da paciente.

\section{CASO CLÍNICO}

Paciente de 24 anos de idade, sexo feminino, procurou atendimento na clínica de prótese da Universidade Nilton Lins apresentando dores orofaciais na região da ATM e nos músculos da mastigação. Durante a anamnese, foi realizado o questionário RDC/TMD (Research diagnostic criteria for temporomandibular disorders, Critérios diagnósticos para pesquisa em DTM), apresentando resultados suscetíveis à disfunção.

Ao exame clínico extraoral, durante a palpação, a mesma apresentou 'pontos de gatilho' em ambos os lados no 
feixe superficial do músculo masseter, no esternocleidomastóideo, na face externa do músculo temporal, no $1 / 3$ superior do trapézio, limitação na abertura bucal máxima. Ao examinar a ATM percebeu-se a presença de estalido, crepitação e deslocamento de disco com redução em ambos os lados, além de desvio mandibular nas lateralidades direita e esquerda e na abertura e fechamento bucal.

Ao exame clínico intraoral constatou-se que a paciente possuía oclusão classe III de Angle, com mordida anterior 'topo a topo', ausência do elemento 13, resultando na movimentação de lateralidade direita com o elemento $14 \mathrm{e}$ desgastes dentários (Figura 1).

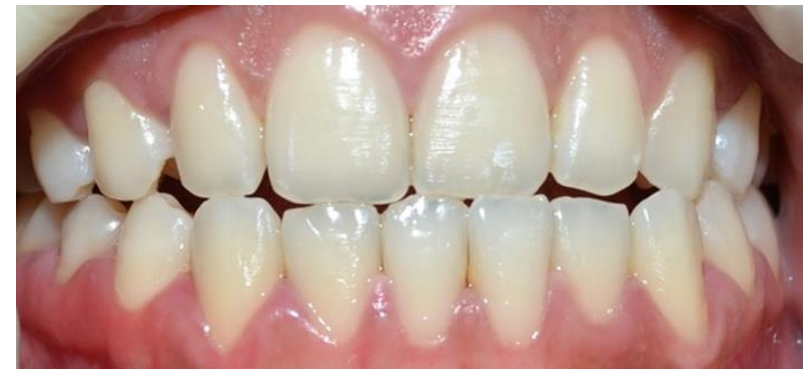

Figura1: Aspecto clínico inicial.

Para uma análise mais minuciosa dos movimentos mandibulares foi realizada a moldagem de diagnóstico com alginato (Hydrogum, Zhermack, Itália), confecção dos modelos de estudo superior e inferior (Figura 2) e montagem no articulador semi-ajustável (ASA), a fim de se obter um diagnóstico preciso e elaborar um plano de tratamento adequado.

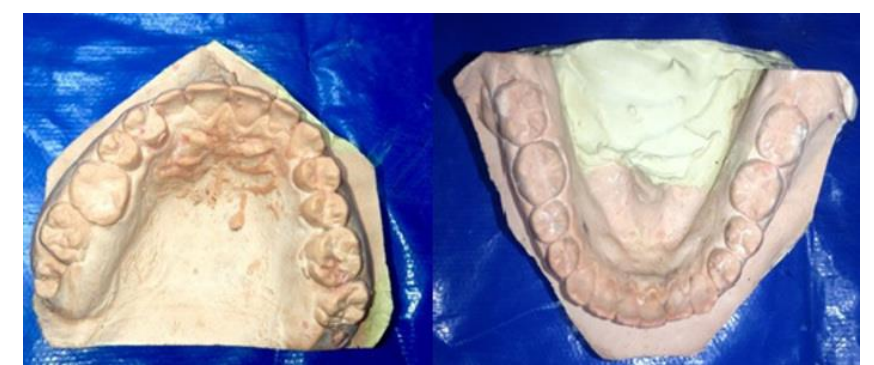

Figura 2: Modelos de estudo superior e inferior.

Após a montagem dos modelos em ASA (Figura 3), foi confeccionado um dispositivo anterior funcional (DAF) ${ }^{12}$ com resina acrílica autopolimerizável (Jet, Clássico, Brasil) para obtenção de registro gráfico dos movimentos mandibulares.

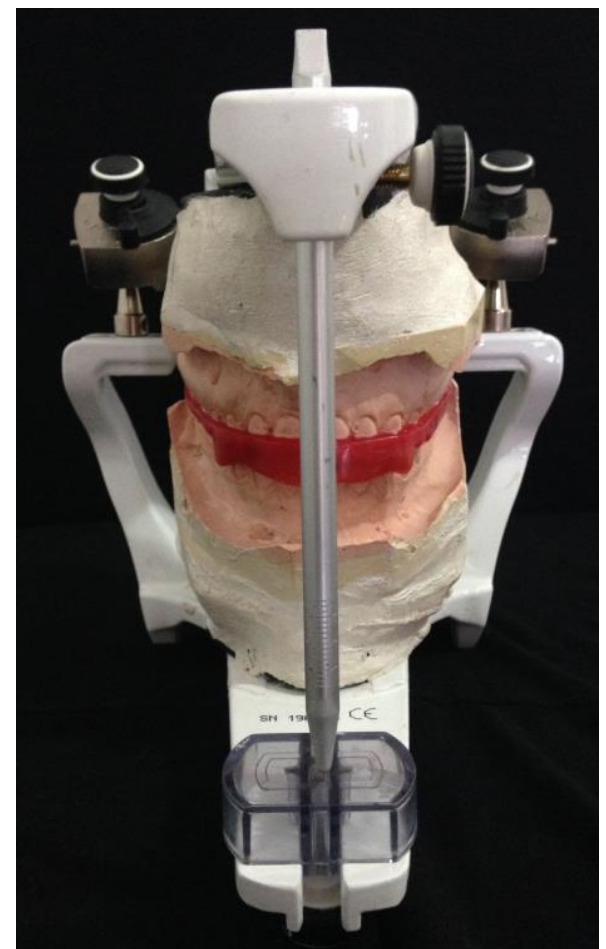

Figura 3: Modelos montados em ASA

A parte A foi reembasada com godiva na região de dentes superiores e a parte B em dentes inferiores (Figura 4). Após isso, solicitou-se à paciente que realizasse os movimentos de lateralidade para ambos os lados e protrusão, onde foi notada a presença de desvios.

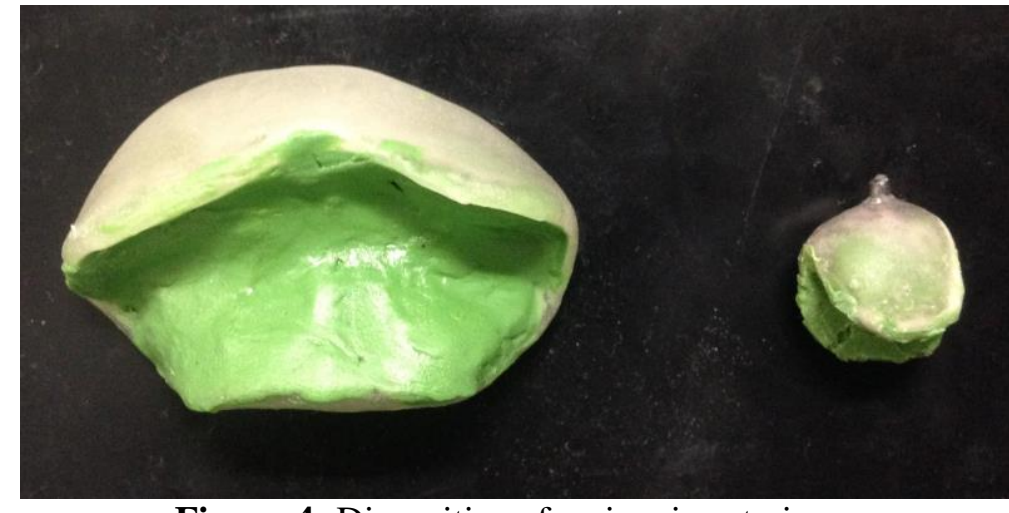

Figura 4: Dispositivos funcionais anteriores reembasados com godiva.

Iniciou-se a confecção do enceramento utilizando lâmina de cera $\mathrm{n}^{\circ} 7$. A mesma foi dobrada ao meio e plastificada, dando o formato de ferradura, e acomodada ao modelo superior. $\mathrm{O}$ articulador foi então fechado promovendo o registro na cera para posterior escultura da placa. $\mathrm{O}$ excesso de cera foi removido, deixando apenas o fundo das cavidades formadas pelas cúspides e incisais dos dentes inferiores (Figura 5). Foi promovido um espaço interoclusal através da interposição entre os arcos de uma placa de acetato de $0,6 \mathrm{~mm}$ (Bio Art, Brasil). Confeccionou-se o guia de desoclusão lateral (guia canino) com intuito de proteger a região láterodistal do disco articular ${ }^{10}$.

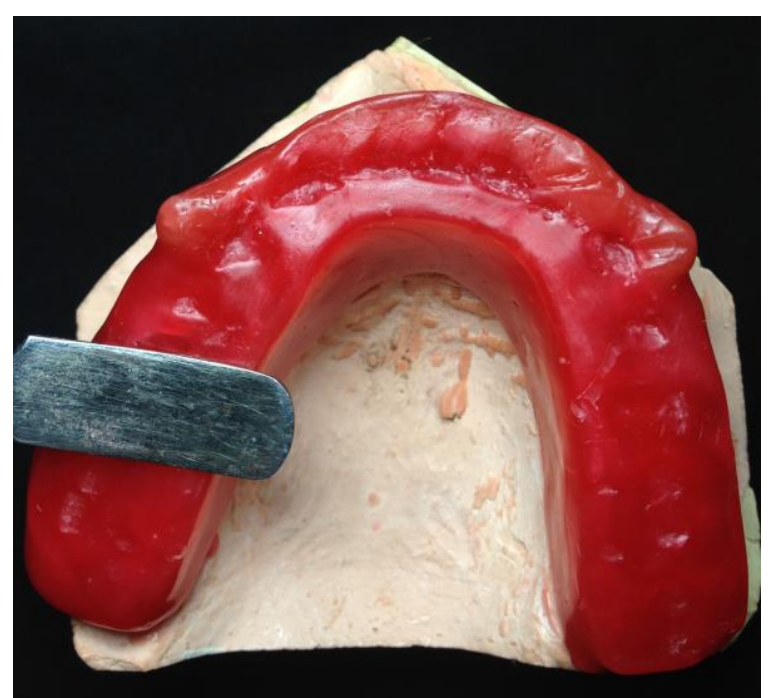

Figura 5: Placa de cera $\mathrm{n}^{\circ} 7$ esculpida após registro oclusal

Após a escultura da placa, procedeu-se a inclusão da mesma de maneira convencional (Figura 6). Foi realizada a remoção da cera, acrilização com resina termopolimerizável (VIPI Cril Plus, VIPI, Brasil) (Figura 7) e a prensagem. Após 12 horas de polimerização a mufla foi aberta e o modelo removido. Em seguida foi realizado o acabamento da placa oclusal.

Para o ajuste em boca, primeiramente foram removidos os contatos prematuros mais grosseiros, seguido do refinamento com auxílio de duas pinças e carbono (Accu Film, Parkell, EUA) fazendo-se os movimentos de lateralidade e protrusiva (Figura 8). Os desgastes foram realizados pela broca maxcut e peça reta, até que se alcançasse a posição miocêntrica da paciente.

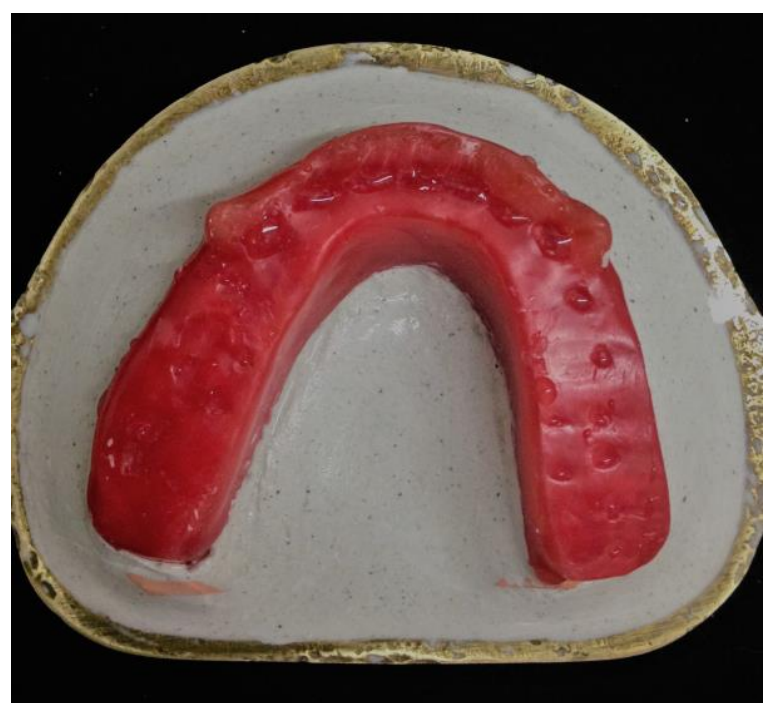

Figura 6: Placa de cera $n^{\circ} 7$ sobre o modelo na mufla. 


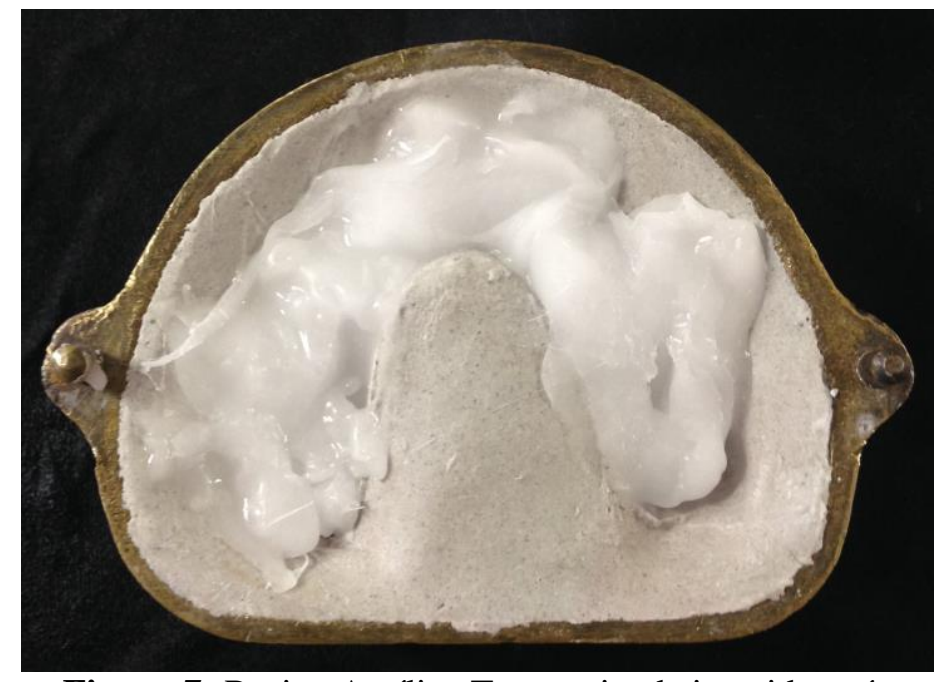

Figura 7: Resina Acrílica Termoativada inserida após a eliminação da cera.

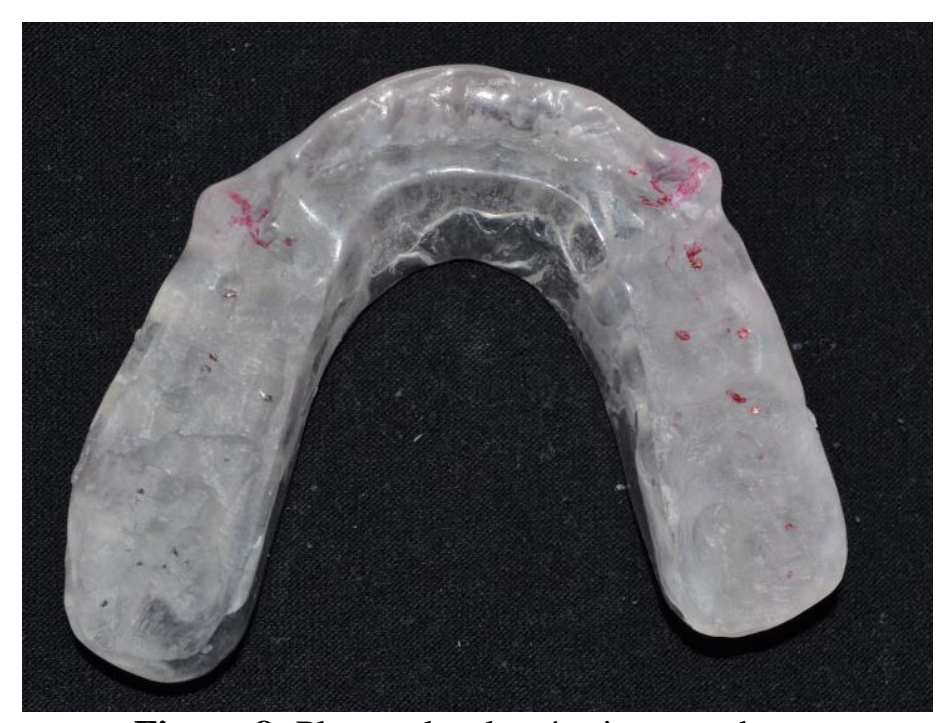

Figura 8: Placa oclusal após ajuste em boca.

Na posição de RC houve o contato de toda superfície da placa com as cúspides vestibulares inferiores e na lateralidade a desoclusão foi realizada pelo canino. A placa oclusal se apresentou perfeitamente adaptada, com estabilidade e retenção.

Após o polimento (Figura 9) a placa foi entregue à paciente para o uso noturno e foram dadas as instruções de higiene (Figura 10). Foram marcados retornos semanais e outro após 45 dias, a fim de se verificar os resultados do tratamento e fazer novos ajustes, caso necessário ${ }^{6}$.

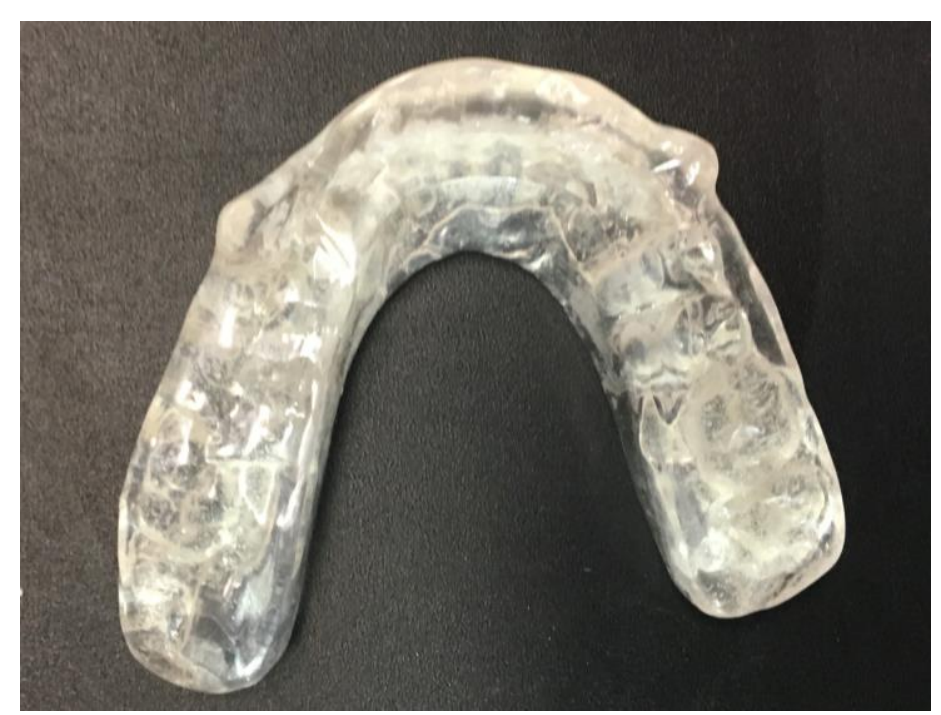

Figura 9: Placa oclusal polida.

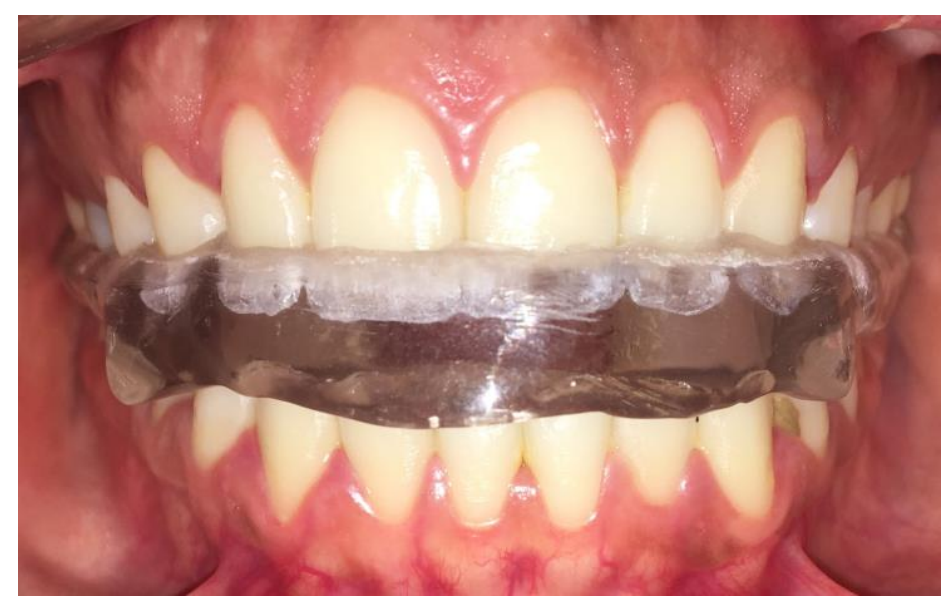

Figura 10: Placa oclusal instalada em boca.

\section{DISCUSSÃO}

As DTMs constituem um assunto de grande interesse na atualidade, devido ao comprometimento na qualidade de vida dos pacientes que sofrem com as dores faciais ${ }^{13}$. É considerada a principal causa de dor não dentária na região orofacial ${ }^{14}$ e afeta primeiramente a musculatura mastigatória, onde as alterações estruturais da ATM tornam os mesmos extremamente tensos. De maneira geral, a idade parece ser uma variável, com maiores prevalências em mulheres com idades entre 20 e 40 anos $^{3,11,13,15}$.

No presente caso clínico, a paciente de 24 anos está dentro da estatística citada e, após a análise dos modelos de estudo no ASA, juntamente com as informações obtidas nos exames intraorais, extraorais e anamnese, o diagnóstico foi DTM associada ao bruxismo cêntrico.

Como se trata se uma disfunção com etiologia de caráter multifatorial, seu diagnóstico e tratamento são complexos, podendo ser citado, entre outros, uso de placas oclusais $^{6}$, fisioterapia ${ }^{7}$, terapia com psicólogo ${ }^{16}$, acupuntura ${ }^{17}$, uso de toxina botulínica ${ }^{18}$, laser de baixa intensidade ${ }^{19}$ e ajuste oclusal $^{20}$.

Os objetivos do tratamento em pacientes com DTMs incluem reduzir a dor, reduzir a sobrecarga adversa e restaurar a função, possibilitando ao paciente retomar as atividades diárias normais ${ }^{4}$, assim, a associação de duas ou mais terapias pode ser indicada.

O tratamento proposto para a paciente do caso apresentado foi o uso de uma placa oclusal superior rígida, pois as placas estabilizadoras de resina acrílica melhoram as desarmonias e possíveis desvios dos movimentos mandibulares, reposicionam a mordida em relação cêntrica, favorecendo uma posição articular confortável, reposicionam o côndilo, relaxando a musculatura mastigatória e suavizando a dor ${ }^{2,6,21,22}$. Seu uso promove reposicionamento condilar, transferindo a carga para diferentes áreas dos tecidos articulares, alternando o comportamento do apertamento $^{14,23,24}$.

Por ser uma disfunção de caráter multifatorial, várias situações podem contribuir para o seu aparecimento, dentre essas sabe-se que as alterações posturais na coluna cervical podem afetar os músculos do sistema estomatognático e causar DTMs ${ }^{25}$. Por essa razão, exercícios à musculatura cervical através do treinamento postural na abordagem do paciente com DTM têm sido aplicados ${ }^{26}$. Entretanto, apesar do treinamento postural ser uma abordagem fisioterápica comum, sua relação com as DTMs ainda não é totalmente compreendida e necessita de mais estudos ${ }^{4,27}$.

Outro tratamento que vem sendo bastante utilizado para diversas situações na área odontológica é o laser de baixa potência, que é uma terapia não invasiva, e que contribui para o alívio das dores mioarticulares e regeneração tecidual, sendo benéfica no tratamento das DTMs ${ }^{19,25,28}$. Porém, os estudos não são conclusivos com relação ao tipo, frequência e durações das radiações com laser e ainda não há uma padronização de tratamento para avaliar a sua real efetividade ${ }^{4,14}$.

Com relação aos tratamentos irreversíveis, o ajuste oclusal foi pesquisado e não apresentou superioridade, em estudos controlados, sobre as terapias reversíveis, por isso é uma opção que deve ser considerada com bastante critério no tratamento primário das DTMs ${ }^{20}$.

A cirurgia de ATM é outra opção irreversível e que pode ser eficaz para distúrbios articulares específicos, porém exige procedimentos mais complexos e pode gerar complicações, principalmente envolvendo a inervação local e o nervo facial ${ }^{29}$. Devido à prevalência de fatores contribuintes comportamentais, psicossociais e por haver diversas abordagens não cirúrgicas, deve-se analisar cada caso para a indicação dessa terapia, devendo o procedimento cirúrgico ser evitado nos casos de pacientes assintomáticos, com 
depressão, com bruxismo noturno intenso, onde não se consiga restabelecer um controle adequado dos fatores musculares e realizado por razões preventivas ${ }^{4}$.

Entre os vários tipos de tratamentos disponíveis, a placa oclusal é uma escolha viável para os casos iniciais de DTM por não ser irreversível e nem invasivo, ter um preço acessível e principalmente por apresentar resultados satisfatórios ${ }^{4,14}$. Assim, optou-se pela utilização da placa oclusal para diminuir os sintomas associados à DTM e atividade parafuncional da paciente, havendo remissão da sintomatologia dolorosa.

\section{CONCLUSÃO}

O tratamento da DTM com placa oclusal estabilizadora rígida foi satisfatório, pois houve redução da intensidade e frequência da dor, com melhora na qualidade de vida da paciente.

\section{REFERÊNCIAS}

1. Uemoto L, Macedo MEG, Alfaya TA, FN Souza, Barcelos R, CVD Gouvêa. Impact of supportive therapy for otological changes in patients with temporomandibular joint disorders. Rev Dor. 2012; 13(3):208-12.

2. Becker AB. Efeito das placas estabilizadora rígida, resiliente e não oclusiva na redução dos sinais e sintomas de pacientes com desordens de origem muscular: revisão de literatura [dissertação]. Araraquara: Faculdade de Odontologia de Araraquara; 2008.

3. Ferreira CLP, Silva MAMR, Felício CM. Signs and symptoms of temporomandibular disorders in women and men. Codas. 2016; 28(1):17-21.

4. Leeuw R. Disfunção temporomandibular. In: Leeuw R. Dor orofacial: guia de avaliação, diagnóstico e tratamento. 4. ed. São Paulo: Quintessence; 2010.

5. Ries LGK, Graciosa MD, Medeiros DL, Pacheco SCDS, Fassicolo CE, Graefling $\mathrm{BCF}$ et al. Influence of craniomandibular and cervical pain on the activity of masticatory muscles in individuals with temporomandibular disorder. Codas. 2014; 26(5):389-94.

6. Nishimori LE, Martins JR, Marson FC, Sábio S, Silva CO, Corrêa GO. Utilização das placas oclusais em resina acrílica no auxílio do tratamento de DTMs: revisão de literatura. Uninga Review. 2014; 17(1):59-64.

7. Torres F, Campos LG, Fillipini HF, Weigert KL, Vecchia GFD. Efeitos dos tratamentos fisioterapêutico e odontológico em pacientes com disfunção temporomandibular: revisão de literatura. Fisioter Mov. 2012; 25(1):117-25.

8. Gil-Martínez A, Grande-Alonso M, López-de-UraldeVillanueva I, López-López A, Fernández-Carnero J, La Touche R. Chronic temporomandibular disorders: disability, pain intensity and fear of movement: research article. J Headache Pain. 2016; 17(1): 103.

9. Portero PP, Kern R, Kusma SZ, Grau-Grullón P. Placas oclusais no tratamento da disfunção temporomandibular (DTM): revisão de literatura. Rev Gestão \& Saúde. 2009; 1(1):36-40

10. Schiffman E, Ohrbach R, Truelove E, Look J, Anderson G, Goulet JP et al. Diagnostic criteria for temporomandibular disorders (DC/TMD) for clinical and research applications: recommendations of the international RDC/TMD consortium network and orofacial pain special interest group. J Orofac Pain Headache. 2014; 28(1):6-27.

11. Sena MF, Mesquita KSF, Santos FRR, Silva FWGP, Serrano KVD. Prevalence of temporomandibular dysfunction in children and adolescents: literature review. Rev Paul Pediatr. 2013; 31(4):538-45.
12. Zanatta EM, Pedro CMB, Seraidarian PI. Dispositivo anterior funcional para localização e registro de relações mandibulo-maxilares. Rev Assoc Paul Cir Dent. 1996; 50(2):133-7.

13. Liu HX, Liang QJ, Xiao P, Jiao HX, Gao Y, Ahmetjiang A. The effectiveness of cognitive-behavioural therapy for temporomandibular disorders: systematic review. J Oral Rehabil. 2012; 39(1):55-62.

14. Okeson JP. Tratamento das desordens temporomandibulares e oclusão. In: Okeson JP. 7. ed. Rio de Janeiro: Elsevier; 2013.

15. Blanco-Aguilera A, Blanco-Hungria A, BiedmaVelazquez L, Serrano-del-Rosal R, Gonzalez-Lopez L, Blanco-Aguilera E et al. Application of an oral healthrelated quality of life questionnaire in primary care patients with orofacial pain and temporomandibular disorders. Med Oral Patol Oral Cir Bucal. 2014; 19(2):127-35.

16. Zavanelli AC, Rezende MCRA, Santos-Neto OM, Fajardo, RS. Integração da Psicologia e Odontologia na DTM: revisão sistemática. Arch Health Invest. 2017; 6(11):530-4.

17. Florian MR, Meirelles MPMR, Sousa MLR. Disfunção temporomandibular e acupuntura: uma terapia integrativa e complementar. Odontol Clín-Cient. 2011; 10(2):189-92.

18. Bicalho MB, Delgado FP, Borini CB. Toxina botulínica no tratamento da disfunção temporomandibular. Políticas e Saúde Coletiva. 2015; 1(2):153-61.

19. Catão MHCV, Oliveira PS, Costa RO, Carneiro VSM. Avaliação da eficácia do laser de baixa intensidade no tratamento das disfunções têmporo-mandibular: estudo clínico randomizado. Rev CEFAC. 2013; 15(6):1601-8.

20. Ossovski CMB. Tratamento de disfunções temporomandibulares através de ajuste oclusal: relato de caso [Trabalho de Conclusão de Curso - Graduação]. Londrina: Universidade Estadual de Londrina; 2012.

21. Almilhatti HJ, Camparis CM, Bönecker G, Ribeiro RA. Como aumentar o índice de sucesso no tratamento com placas oclusais miorrelaxantes: revisão de literatura. JBA. 2003; 2(8):340-3.

22. Lima DG, Oliveira DWD, Oliveira ES, Gonçalves PF, Flecha OD. Placas estabilizadoras em pacientes portadores de DTM: relato de dois casos. Rev Bras Odontol. 2016; 73(3):261-4.

23. Pficer JK, Dodic S, Lazic V, Trajkovic G, Milic N, Milicic B. Occlusal stabilization splint for patients with temporomandibular disorders: meta-analysis of short and long term effects. Plos One. 2017; 12(2):e011296.

24. Zuim PRJ, Garcia AR, Costa PS, Turcio KHL, Zaze CA. Como fazer placas interoclusais? Alguns aspectos a se considerar: revisão de literatura. Rev Odontol Araçatuba. 2008; 29(2):40-5.

25. Oliveira KB, Pinheiro ICO, Freitas DG, Gualberto HD, Carvalho NAA. A abordagem fisioterapêutica na disfunção da articulação temporomandibular: revisão da literatura. Med reabil. 2010; 29(3):61-4.

26. Calixtre LB, Grüninger BL, Haik MN, AlburquerqueSendín F, Oliveira AB. Effects of cervical mobilization and exercise on pain, movement and function in subjects with temporomandibular disorders: a single group prepost test. J Appl Oral Sci. 2016; 24(3):188-97.

27. Moraes AR, Sanches ML, Ribeiro EC, Guimarães AS. Therapeutic exercises for the control of temporomandibular disorders. Dental Press J Orthod. 2013; 18(5):134-9.

28. Assis TO, Soares MS, Victor MM. O uso do laser na reabilitação das desordens temporomandibulares. Fisioter Mov. 2012; 25(2):453-9.

29. Grossmann E, Grossmann TK. Cirurgia da articulação temporomandibular. Rev Dor. 2011; 12(2):152-9. 


\section{CONFLITO DE INTERESSES}

Os autores declaram não haver conflitos de interesse.

\section{AUTOR PARA CORRESPONDÊNCIA}

Gisely Naura Venâncio ginaura@gmailcom

Submetido em 12/03/2018

Aceito em 11/05/2018 